\title{
Preparation of Chitosan-Silver Nanoparticles in Nonaqueous Medium under Heating
}

\author{
A. B. M. Nazmul Islam \\ Department of Natural Sciences, Faculty of Science and Information Technology, Daffodil \\ International University, Dhaka- 1207, Bangladesh \\ E-mail address: nazmul.acce.26@gmail.com
}

Keywords: Chitosan, chitosan-silver nanoparticles, dimethylformamide, sodium dodecyl sulfate (SDS), grain-rotation induced grain coalescence (GRIGC).

\section{ABSTRACT}

Chitosan-silver nanoparticles are prepared in nonaqueous mediup. In this wh odium dodecyl sulfate (SDS) was introduced into the dimethylformamide (D so atnon du ng silver reduction from solution of its precursor salt $\mathrm{AgNO}_{3}$, acting as $\mathrm{ab}$, age to prevent aggregation of silver nanoparticles, while chitosan is used as the lid suppo enbedded silver particles therein, resulting in chitosan-silver (CS-Ag) nanopa iclo suspensi $n$ in the medium. The reaction started as homogeneous system which turned iv leterog ous with the formation of particles. The properties of CS-Ag nanoparticles are studied un two different salt concentrations and characterized by atomic forc microscopy (AFM), scanning electron microscopy (SEM), fourier transform infrared $\mathrm{sp}$ troscopy (TIR) and ultraviolet-visible spectroscopy (UV-Vis). Wide particle size distribution syntho szed nanoparticles depicts that concentration of $\mathrm{AgNO}_{3}$, which is responsi for the stology, stability and particle size distribution, should be optimized, suggesting a the concentration is favorable.

\section{INTRODUCTION}

There has been a tremend inc ase in the number of patients affected by diseases like diabetes and cancer during t'ast aae. so, it has become the major focus of researchers to synthesize a compound th vill be use foy the monitoring of certain elements that are present in excess quantities in the floo hile used $n$ the form of biosensors, at the same time being useful for the treatment asseases ce cancer. Chitosan (CS) is a polysaccharide composed of glucosamine and N-acetyl glucosa ne linked with a $\beta, 1-4-$ glycosidic linkage[1]. Chitosan is a biopolymer $w^{\prime}$ is compatible and can be degraded by enzymes in human body, the degradation proa are no toxic. Commercial Chitosans are semi-crystalline polymers and crystallini ays a ppo tant role in adsorption efficiency [2]. Chitosan is a polymer which exhibi a bro d-spectro of antimicrobial activity by binding to the negatively charged bacterial cell Wa 11 attachment to the DNA, inhibiting its replication [3, 4]. For the improvement of bioacti on chitosan, it is often combined to other bioactive materials, such as drugs.

Silver of nanoparticles have high therapeutic potential and exhibit good antimicrobial activity. Ag hanoparticles have a wide range of antimicrobial activities and exhibit high performance even at a very low concentration. Ag nanoparticles have been identified to possess good potential for the treatment of cancer [5]. But the major disadvantage of using silver alone is that it is not specific at targeting the cancer cells and also it is toxic to the normal cells when exposed for a longer time when the size of silver is used $>20 \mathrm{~nm}$ [6], CS-Ag nanocomposite is one of the rare composite materials that is seen to possess a capability of being used as a biosensor as well as in the treatment of cancer as the chitosan present in the nanocomposite is very specific to the cancer cells. It prolongs the action of silver on the affected cells while preventing the normal cell from the effect of silver. One more advantage of this nanocomposite is that it is biodegradable i.e., it can be degraded by the enzymes present in the body making it suitable for the treatment of 
cancer. Apart from the treatment of cancer, the nanocomposite also possesses good antimicrobial [7] and biosensing activity.

Sodium dodecyl sulfate (SDS) is introduced into the amide solution at the start of reaction or continuously during metal reduction [8]. SDS plays a twofold role it acts as a stabilizing agent to prevent aggregation of metal nanoparticles. In addition, SDS can be adsorbed on the surface of specific crystal faces and serve as shape control agent. In this work, we proposed the synthesis method by reducing $\mathrm{AgNO}_{3}$ in dimethylformamide (DMF) with chitosan by varying concentration for the preparation of silver nanoparticles. The influence of the salt concentration on the optical properties, structures and morphologies of CS-Ag nanoparticles was characterized by the atomic force microscopy (AFM), ultraviolet-visible spectroscopy (UV-Vis), scanning electron microscopy (SEM) and fourier transform infrared spectroscopy (FTIR).

\section{EXPERIMENTAL}

Materials

Prawn shell was collected from Rupsa ferry ghat, Rupsa, Khulna, Bang lesh it igure Sodium hydroxide pellet, hydrochloric acid and absolute alcohol were obtai d Merc I lia, acetone was obtained from Merck Germany, potassium bromide was obtai cd from S a ul a USA, DMF from Fisher scientific UK, SDS from Fulka analytical Switze ia and silve, itrate form UniChem China.

3. METHO Extraction aitin, prown shell

Colle a pra $\mathrm{h}$ shell was washed with hot water and then dried in an oven at $105^{\circ} \mathrm{C}$ for 72 hours. Dried $P$ as grounded. Deproteinized was carried out with $1 \mathrm{~N} \mathrm{NaOH}$ at boiling temperatu 4 hours in prawn shell: $\mathrm{NaOH}$ ratio of $1: 16 \mathrm{w} / \mathrm{v}$, followed by neutralization. Residue was deminera ed with $1 \mathrm{~N} \mathrm{HCl}$ at boiling temperature for 4 hours in material:HCl ratio of 1:13 w/v. Excess acid was neutralized [9].

Conversion of chitin into chitosan

Deacetylation of chitin was carried out using $40 \% \mathrm{NaOH}$ (Chitin: $\mathrm{NaOH}=1: 20$, w/w) at boiling temperature for 3.5 hours. After deacetylation solid was separated from alkali and extensively washed with distilled water to remove traces of alkali. Resultant solid was dried in a vacuum oven at $60{ }^{\circ} \mathrm{C}$ for 24 hours. The material obtained in this step is known as chitosan. Chitosan was grounded and placed in a desiccator for storage [9]. 
Preparation of chitosan-silver nanoparticles

$0.05 \mathrm{~g}$ of chitosan was dissolved in $20 \mathrm{~mL} 10 \mathrm{~N} \mathrm{HCl}$ solution and used as stock solution. $5 \mathrm{~mL}$ stock solution, $5 \mathrm{~mL}$ DMF and $0.05 \mathrm{~g}$ of SDS were mixed for $30 \mathrm{~min}$ using magnetic stirrer to get homogeneous solution. $4.90 \mathrm{mM}$ silver nitrate salt was added in $10 \mathrm{~mL}$ DMF and mixed for $30 \mathrm{~min}$ using magnetic stirrer to get homogeneous solution. The total volume of solution was $20 \mathrm{~mL}$ and final concentration of $\mathrm{AgNO}_{3}$ was $2.45 \mathrm{mM}$ and these placed in a three neck round bottom flask. Out of three neck of the round bottom flask, one neck was kept for solution upload, the other neck was used for thermometer and the middle one was used for condenser setup.

When the effect of concentration of Ag precursor salts was examined, the concentration of $\mathrm{AgNO}_{3}$ was changed from $2.45 \mathrm{mM}$ to $5 \mathrm{mM}$ for the preparation of CS-Ag nanoparticles. The Ag/SDS molar ratio was kept at the same values as those used at initial condition. The solution tamnerature was increased from room temperature to $120^{\circ} \mathrm{C}$ by heating for $10 \mathrm{~min}$.

Analyses of size and component of products

For AFM (Park Systems, XE-70, South Korea), and SEM (Hitachi, modeh S 3. N VPS Japan) observations CS-Ag particles were obtained from ethanol solut on by cen "giv g the colloidal solution at 15,000 rpm for $30 \mathrm{~min}$ in three times. Fourier trans $f^{\prime} \mathrm{m}$ infr $\mathrm{d}$ (Pa nElmer, Spectrum 100, USA) spectroscopy and Ultraviolet-visible (Shin dzu -1650 PC, Japan) spectroscopy were also measured.

\section{RESULT AND DISCUSSION}

AFM observation

Freshly prepared nanoparticles were centrifuged with for film and subsequent dried in open air dry at roon Systems, XE-70, South Korea) images of CS-Ag concentrations such as $2.45 \mathrm{mM}$, and $5 \mathrm{mM}$.

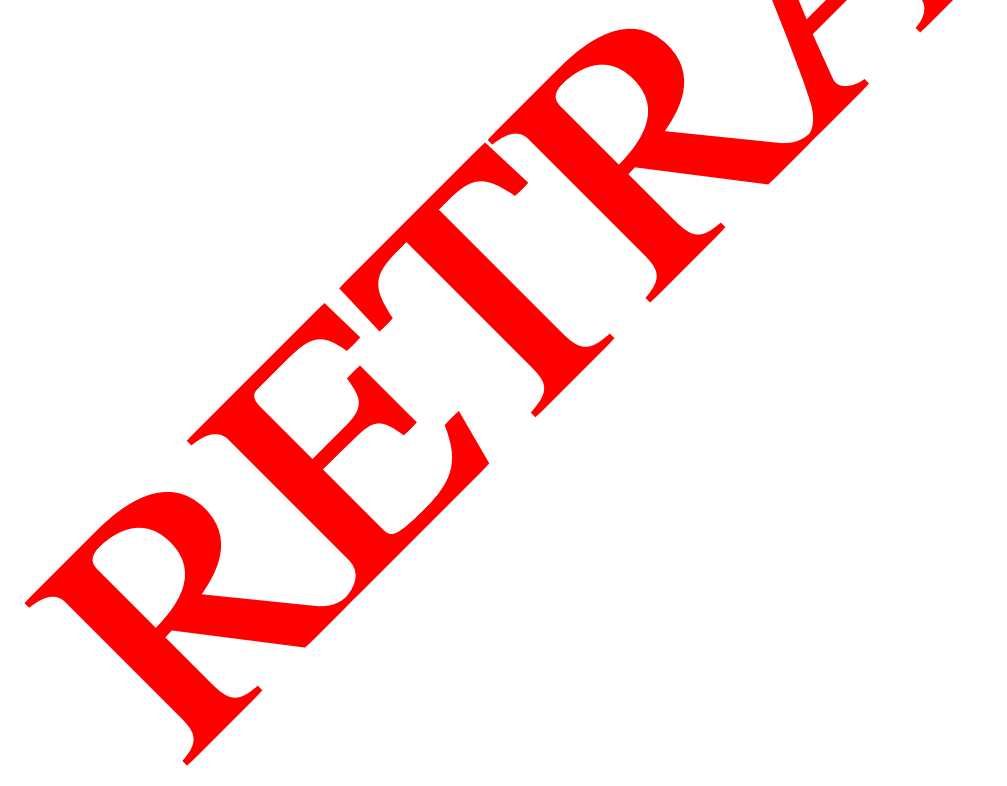

anol, follo ed by casting onto glass slide

mperatur . Figure 2 depicts AFM (Park 1. cles with different metal ion 

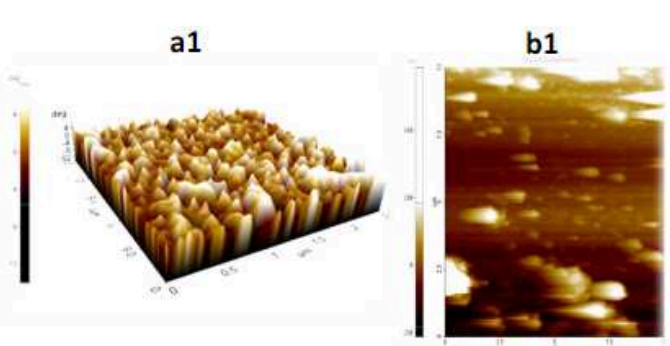

$2.45 \mathrm{mM}$

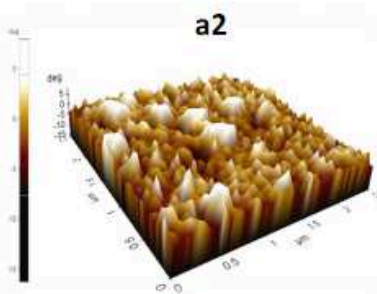

b2

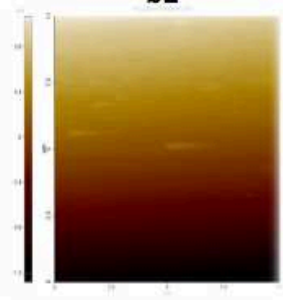

$5 \mathrm{mM}$

Figure 2. a1-c2 represent topographical height and frequency histogram CS-Ag nt artic/s using $2.45 \mathrm{mM}$ and $5 \mathrm{mM} \mathrm{AgNO}_{3}$ salt respectively.

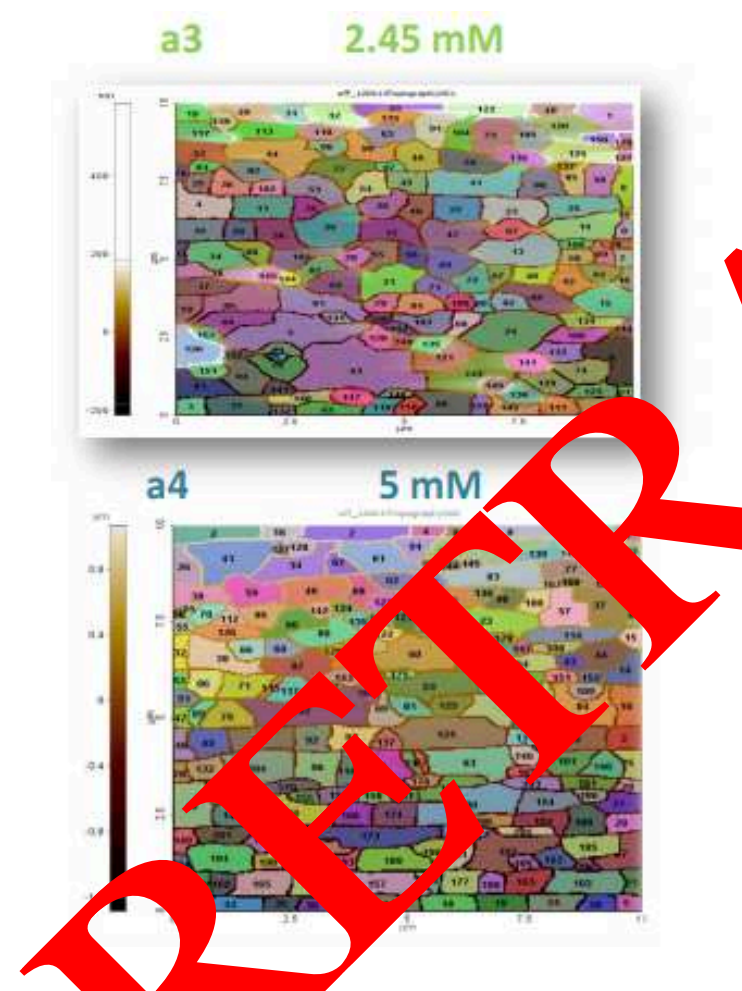

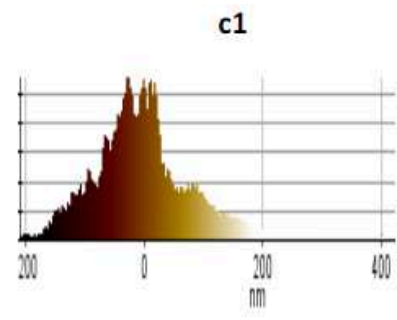

c2
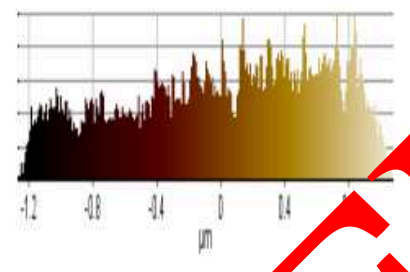

Fig 3. as-os and a4-b4 reveal the grain size of $2.45 \mathrm{mM}$ and $5 \mathrm{mM}$ of CS-Ag nanoparticles respec
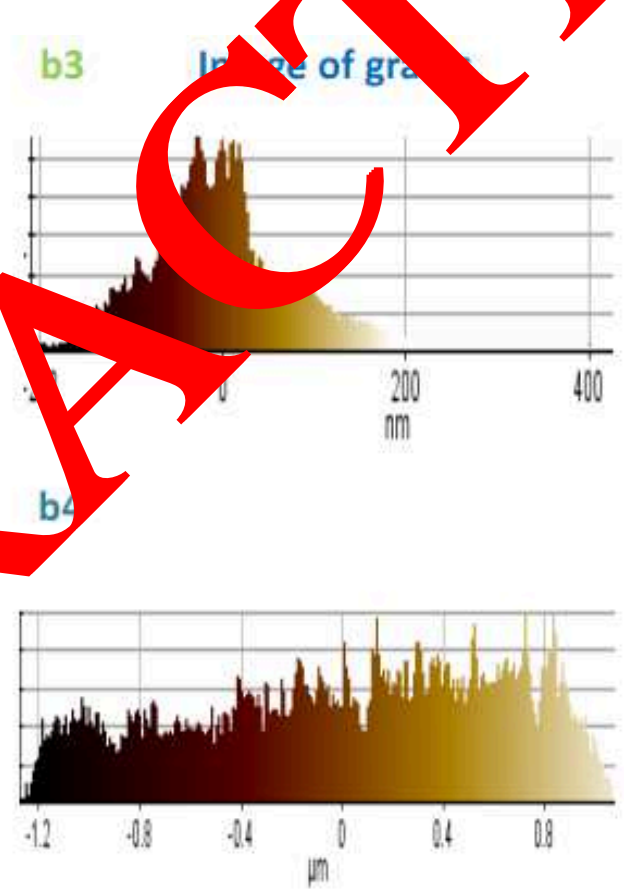

\section{Effect of precursor salt concentration}

The effect of precursor salt concentrations is examined by changing silver nitrate concentrations from $2.45 \mathrm{mM}$ to $5 \mathrm{mM}$ to prepare CS-Ag particles. The molar ratio of Ag salt and SDS was kept constant for the preparation of CS-Ag particles in reaction medium. 


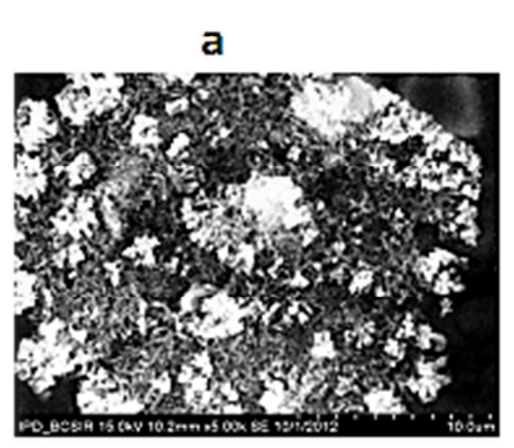

CS

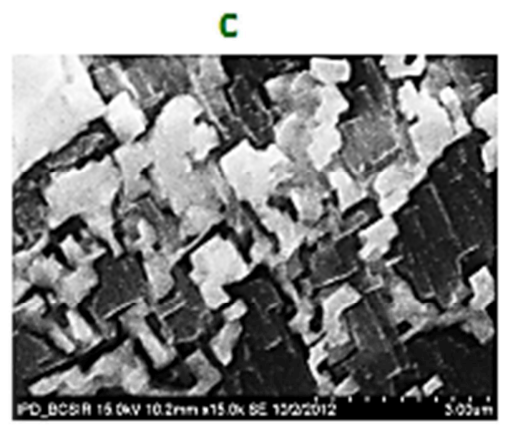

CS-Ag, $5 \mathrm{mM}$

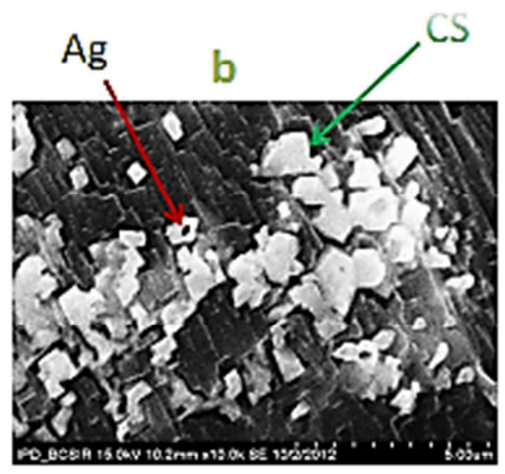

$\mathrm{CS}-\mathrm{Ag}, 2.45 \mathrm{mM}$

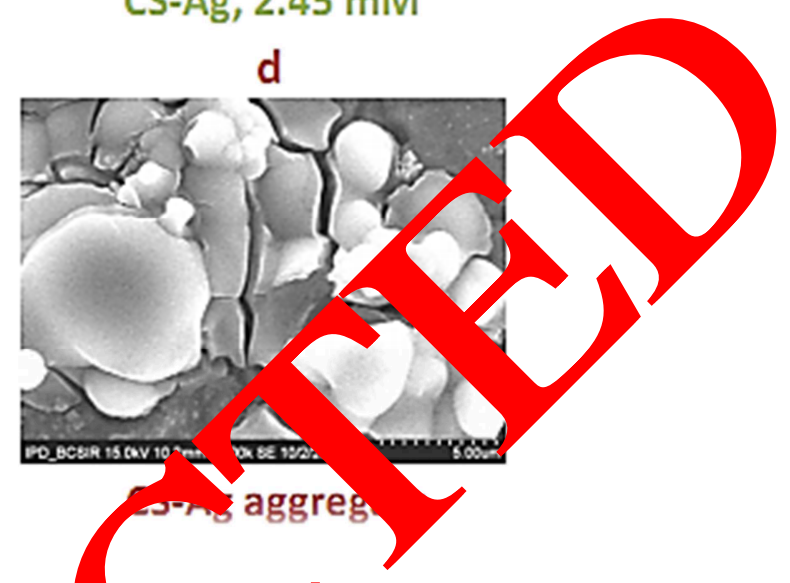

Figure 4. a, b, c and d represent SEM micrograph of fres 1 prepared chi psan, Cs-Ag nanoparticles for $2.45 \mathrm{mM}, 5 \mathrm{mM}$ and aggregated nanoparticles under heatir

Figures 2a1-c2 show AFM data at 2.45, and $5 \mathrm{~m} 1 \mathrm{c} / \mathrm{As}_{3}$ salt solution after heating for $10 \mathrm{~min}$. In AFM topography, shown in Figures $201-c 1$ top graphical images and frequency histogram depict that the size of the most prepared n nopa cles are $-200 \mathrm{~nm}$ using $2.45 \mathrm{mM}$ salt in Figures $2 \mathrm{~b} 1-\mathrm{c} 1$. At $2.45 \mathrm{mM}$, CS-Ag nanocompe siz distribut on are in the range 1-200 $\mathrm{nm}$ but major products are spherical or plate like CS p. dissolved silver ion is reduced to small (CS-Ag) particles that are abundan disperse solution shown in Figures 3a3-b3. At $5 \mathrm{mM} \mathrm{CS-Ag}$ nanocomposites size di rib. are in t range $1-800 \mathrm{~nm}$, no significant shape change occur in CS-Ag particles but ajor proc are larger and nearly spherical and/or bar like CS-Ag particles produced that are oigger than 2.4. $\mathrm{M}$ M salt concentration in Figures 3a4-b4. These results imply that shape, and e dis zutions of the product depend on the metal ion concentration and lower $\mathrm{AgNO}_{3}(2.45 \mathrm{mk}$ oncentra on than $5 \mathrm{mM}$ is favorable for the preparation of CS-Ag particles with near herica $\mathrm{nd} / \mathrm{c}_{\mathrm{I}}$ plate like shape. It shows that with increasing salt concentration change n phà angle to differing amount of damping experienced by the probe tip as it rusters across

SEM obsu tion:

The surface Shology of synthesized CS-Ag nanocomposite was analyzed using SEM (Hitachi, model-S 3400 N VPSEM, Japan) technique. The SEM image of CS shows that the particles are in the form of bundles with a leaf morphology in Figure 4a. The SEM image of CS-Ag nanoparticles shows nearly spherical or plate like shaped particles in Figure $4 \mathrm{~b}$ for $2.45 \mathrm{mM}$. The Figure $4 \mathrm{~b}$ shows a mixture of CS and Ag wherein the silver nanoparticles are seen to be enveloped by the chitosan polymer indicated in Figure $4 b^{10}$. In case of $5 \mathrm{mM}$ concentration, the synthesized particles are in the form of aggregates or larger in size than that of $2.45 \mathrm{mM} \mathrm{CS}-\mathrm{Ag}$ particles in Figure 4c. It is observed that the silver nanoparticles are embedded in a matrix of chitosan. The morphology of the silver nanoparticles was predominantly nearly spherical or plate like in Figure $4 \mathrm{c}$ and aggregated into larger irregular structure with no well-defined morphology observed in the micrograph of Figure $4 \mathrm{~d}$ under heating or storage. 
FTIR observation:

FTIR observation is measured by using infrared spectrophotometer (PerkinElmer, Spectrum 100, USA). KBr diluted nanoparticles are washed with alcohol and centrifuged to collect particles, subjected to give the FTIR spectra of chitosan and CS-Ag nanoparticles are depicted in Figure 5. The band at 3411 and $3467 \mathrm{~cm}^{-1}$ correspond to $\mathrm{O}-\mathrm{H}$ stretching $\mathrm{H}$-bonded alcohols and phenols of CS-Ag nanoparticles and chitosan itself respectively. The peak at 1666 and $1622 \mathrm{~cm}^{-1}$ correspond to $\mathrm{N}-\mathrm{H}$ bond primary amines of CS-Ag nanoparticles and chitosan itself respectively. The peak at 2835 and $2831 \mathrm{~cm}^{-1}$ correspond to $\mathrm{O}-\mathrm{H}$ stretch carboxylic acids of CS-Ag nanoparticles and chitosan itself respectively. The peak at 1402 and $1383 \mathrm{~cm}^{-1}$ correspond to C-N stretching of aromatic amine group of CS-Ag nanoparticles and chitosan itself respectively. The other bands are of $\mathrm{C}-\mathrm{O}$ stretch alcohols and carboxylic acids observed at characteristics spectral shiftc Therefore the synthesized CS-Ag nanoparticles are surrounded by chitosan having functi nal gi s of amines, carboxylic acids, alcohols and esters.

Figure 5. FTIR spectra o
UV-Visible spectroscopy analysis

The UV-Visible spectral measu ments were ca jed out using UV-Visible spectrophotometer (Shidmadzu, UV-1650PC, Jap (). Sorption spectra recorded is quite sensitive to the formation of silver nanopar cles be e of the fact that silver nanoparticles exhibit an intense absorption peak due to s plasmon onance (SPR). Figure 6 shows the UV-Vis spectra of silver nanoparticles pro ared $\mathrm{n}$ two different concentrations of silver nitrate $(2.45 \mathrm{mM}$, and 5 $\mathrm{mM}$ ) with chitosan $\Omega \mathrm{L}$ ) for $\mathrm{in}$ heating. All spectra exhibit an absorption band in the range of 300-400 nm sith a vpical pla, mon resonance band of CS-Ag nanoparticles. The absorption against wavelen. cu ves at various concentrations is shown in Figure 6. It was noticed that the reduction capacity chito an increased with varying concentration of $\mathrm{Ag}$ salt. As the salt concentrato ncreas ossibly more and more of hydroxyl groups are converted to carbonyl group vair xidation, which in turn reduces more silver ions at a constant chitosan concentration. A singlo with a maximum around $295 \mathrm{~nm}$ was observed in the UV-Vis spectra, which correspona typical SPR of conducting electrons from the surface of silver nanoparticles. The intensity of th absorption of solutions increased with increase in the concentration of $\mathrm{AgNO}_{3}$ up to $5 \mathrm{mM}$ at constant heating time. Ag nanoparticles with nearly spherical morphology are embedded in the chitosan matrix which arises a small peak at around $380 \mathrm{~nm}$ in Figure 6 . As the particles increase in size, the absorption peak usually shifts toward the red wavelength [11]. 


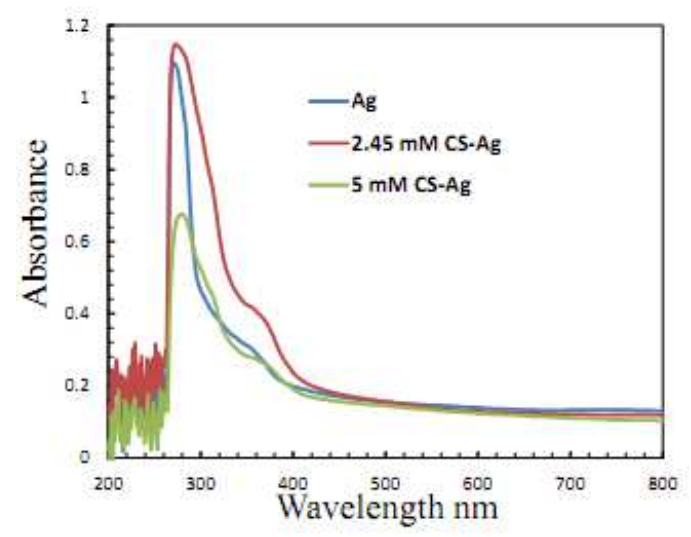

Mechanism

Figure 6:. UV-visible spectra of Ag salt solution, $2.45 \mathrm{mM}$ and $5 \mathrm{mM}$ of CS-Ag nano rticles.

The growth mechanisms of CS-Ag nanostructures under different summarized in Figure 7. In this process a large amount of CS-Ag nopart les an cpared. Mechanism in Figure 7 is the grain-rotation induced grain coalesec (CRIG and fusion mechanism $^{12}$. According to this model, the rotation of grains amon no,ghb grai s results in a coherent grain grain interface which leads to the coalescen f neighb grains via the elimination of common grain boundaries, thus forming a lar $r$ gra In our gase, single crystal, particles having well defined faces were lost after the form of la oolycrystalline spherical particles. Thus, during GRIGC process fusion of po gonal crystals ading to polycrystalline spherical or plate like particles. In the Ostwald-ripeni g mechanism, the atoms from one particle undergo dissolution and then they are transferred to an er particle There is a net atomic transport from the particles with sizes smaller than the average va larg particles. Particles smaller than the average value will shrink or even disappear

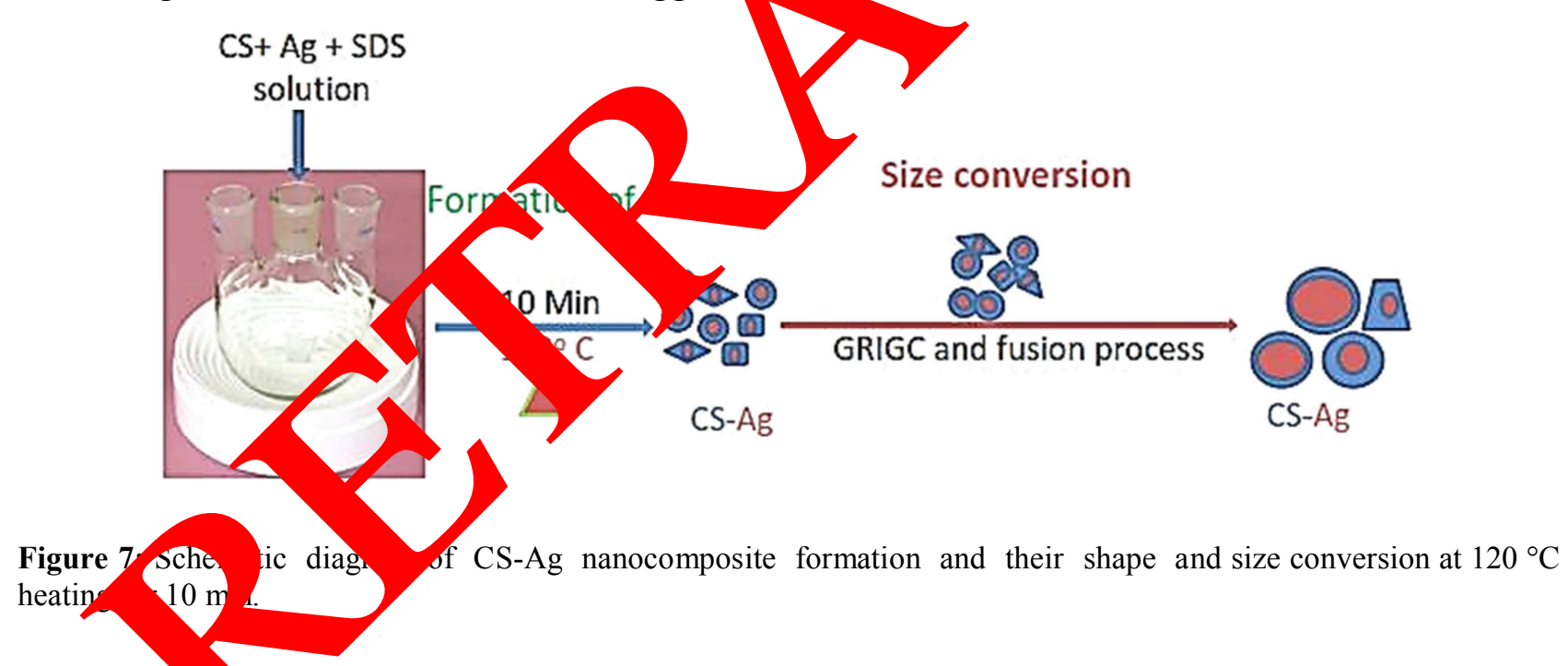

\section{CO YUSION}

Silver n noparticles were successfully introduced into chitosan using non aqueous thermal reduction method. This was confirmed by the maximum surface resonance peak at $295 \mathrm{~nm}$. Topographical heights of the CS-Ag nanoparticles were revealed to be around $295 \mathrm{~nm}$ for most of the particles made from $2.45 \mathrm{mM}$ and $5 \mathrm{mM}$ silver nitrate salt. In SEM micrograph nanoparticles with nearly spherical or plate like morphology are embedded in the chitosan matrix which is arise a small peak at around $380 \mathrm{~nm}$. From FTIR spectra it was revealed that the synthesized nanoparticles are surrounded by chitosans having functional groups of amines, carboxylic acids, alcohols and esters. Wide particle size distribution of nanoparticles synthesized here depicts that the parameter, concentration of $\mathrm{AgNO}_{3}$, which is responsible for the morphology and stability, should be optimized, suggesting lower salt concentration is favorable. 


\section{References}

[1] G Crini, P. M. Badot Application of chitosan, a natural aminopolysaccharide, for dye removal from aqueous solutions by adsorption processes using batch studies. A review of recent literature Prog. Polym. Sci., 33, 1 (2008) 399-447.

[2] TS Trung, WW Thein-Han, NT Qui, C-H Ng, WF Stevens Functional characteristics of shrimp chitosan and its membranes as affected by the degree of deacetylation. Bioresour Technol., 97, 2 (2006) 659-663.

[3] K Kurita, K Tomita, T Tada, SL Nishimura, Ishii S Reactivity characteristics of a new form of chitosan. Polym Bull., 30, 3 (1993) 429-433.

[4] DR Ting, Y Shen Antibacterial finishing with chitosan derivatives and their anlo $_{1}$ icles. Dyeing Finishing, 14, 4 (2005) 12-14.

[5] MI Sriram et al Antitumor of silver nanoparticles in Dalton's lyw yoma cites t mor model. Int. J. Nanomed, 5 (2010)753-762.

[6] R Vaidyanathan, K Kalishwaralal, S Gopalram, S Gurunathan

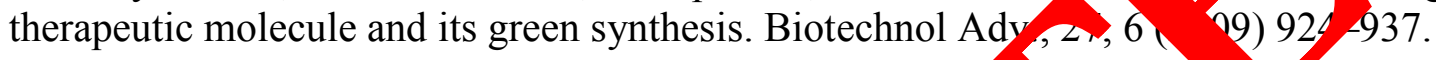

[7] P Sanpui , A Murugadoss, PV Durga Prasad, SS O h, A Cho spadhyaya The antibacterial properties of a novel chitosan-Ag opar composite. Int J Food Microbiol., 124, 7 (2008) 142-146.

[8] CC Chang, HL Wu, CH Kuo and MH Huang Hydrothermal Synthesis of Monodispersed Octahedral Gold Nanocrystals with Five Diffe t Size Rar ges and Their Self-Assembled Structures, Chem. Mater., 20, 24 (2008) 7570- $\lambda$

[9] YT Antonia, Optimization studies on o xtraction from crustaceansolid wastes, M.Sc. Thesis, Department of Food Science a d gral Chemistry, Mc Gill University,
Montreal, Qubee (1991 ) 43-51

[10] K Yoshizuka, Z Lou, K noue Silver-c Aplexed chitosan microparticles for pesticide removal. React Funct Poyin 44 nunony 47-54.

[11] Y Xia and NJ Hal $<$ Shape trolled Synthesis and Surface Plasmonic Properties of Metallic Nanostry ctu. MRSBu, an, 30 (2005) $338-343$.

[12] MJ Alam, M suji, M sunagz Shape Changes from Polygonal Gold Nanocrystals to Spherical sanop articles In aced by Bubbling $\mathrm{N}_{2}$ or $\mathrm{O}_{2}$ Gas in Polyol Synthesis of Gold Nanosty res. Shem. Soc. Jpn., 83, 1 (2010) 92-100.

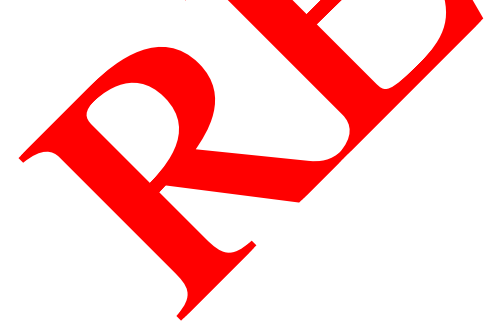

